\section{RMD Open}

Rheumatic \&

Musculoskeletal Diseases

\title{
Understanding the association between skin involvement and joint activity in patients with psoriatic arthritis: experience from the Corrona Registry
}

To cite: Mease PJ, Etzel CJ, Huster WJ, et al. Understanding the association between skin involvement and joint activity in patients with psoriatic arthritis: experience from the Corrona Registry. RMD Open 2019;5:e000867. doi:10.1136/ rmdopen-2018-000867

- Prepublication history for this paper is available online. To view these files, please visit the journal online (http://dx.doi. org/10.1136/rmdopen-2018000867).

Portions of this work have been previously presented at the 2017 ACR/ARHP Annual Meeting, 3-8 November 2017, San Diego, California, Poster No 62600,

'The relationship between the degree of skin involvement and joint activity in patients with PsA: experience from the Corrona Registry.

Received 27 November 2018 Revised 9 March 2019 Accepted 4 April 2019

Check for updates

(C) Author(s) (or their employer(s)) 2019. Re-use permitted under CC BY-NC. No commercial re-use. See rights and permissions. Published by BMJ.

For numbered affiliations see end of article.

Correspondence to Professor Philip J Mease; pmease@philipmease.com

\section{ABSTRACT}

Objective To compare the characteristics of patients with psoriatic arthritis among patient groups stratified by degree of skin and joint involvement, and to evaluate the relationship between skin severity and joint activity. Methods Body surface area (BSA) and Clinical Disease Activity Index (CDAl) at enrolment were analysed. Patient characteristics were stratified by skin severity and joint activity. Baseline patient characteristics, clinical and disease characteristics and patient-reported outcomes were compared. The strength of the relationship of skin severity and joint activity was evaluated using methods for categorical variables ( $\chi^{2}$ test, Cramer's $V$ ) and continuous variables (linear regression).

Results 1542 adult patients in the Corrona Psoriatic Arthritis/Spondyloarthritis Registry enrolled between 21 May 2013 and 20 September 2016 were analysed. Most patients in the BSA $>3 \% / C D A l$ moderate/high subgroup had worse clinical and patient-reported outcomes. A significant $(p<0.001)$ modest association (Cramer's $\mathrm{V}=0.1639$ ) between skin severity and joint activity was observed among all patients at enrolment. Patients with higher skin severity were two times more likely to have higher joint involvement (OR 2.27, 95\% $\mathrm{Cl} 1.71$ to 3.01 ). A significant linear relationship between CDAI and BSA was observed. Effect modification showed this linear relationship was modified by age, gender, insurance, work status, current therapy, Health Assessment Questionnaire, Nail visual analogue scale, minimal disease activity, dactylitis count, patient-reported pain and fatigue.

Conclusion Skin severity is modestly correlated with joint activity, and patients with higher skin severity are two times more likely to have increased joint involvement. Clinicians need to address both skin severity and joint activity in treatment decisions.

\section{INTRODUCTION}

Psoriatic arthritis (PsA) is a chronic, inflammatory condition that presents with cutaneous (involvement of skin and nails), musculoskeletal (arthritis, enthesitis,

\section{Key messages}

What is already known about this subject?

- Limited information is known about the relationships between skin disease severity and joint disease activity in patients with psoriatic arthritis (PsA).

- Studies examining the clinical characteristics of patients with PsA have not found consistent correlations between baseline skin and joint disease measures.

What does this study add?

- Our study found the strength of the relationship between joint disease activity and skin disease severity was modest, yet statistically significant.

- Patients with higher skin disease severity (body surface area $(\mathrm{BSA})>3 \%$ ) were two times more likely to have higher joint disease involvement compared with those with a $\mathrm{BSA}=0 \%$.

How might this impact on clinical practice?

- Clinicians (specifically rheumatologists and dermatologists) need to approach PSA patient care comprehensively addressing both the skin disease severity and joint disease activity in the treatment of these patients.

dactylitis and spondylitis) and extra-articular features (inflammatory bowel disease, uveitis). ${ }^{1-3}$ Psoriasis (PsO) affects up to $3.2 \%$ of the population in the USA, and studies have found up to $30 \%$ of patients with $\mathrm{PsO}$ have PsA. ${ }^{45}$ Compared with the general population, patients with PsA have a lower health-related quality of life (QOL) and experience a higher risk of developing comorbidities including malignancy and cardiovascular disease. Previous studies of patients with PsA have shown that patients with skin involvement have a more significant impairment of QOL measures and an 
increase in overall comorbidities. ${ }^{67}$ Studies examining the clinical characteristics of patients with PsA have not found consistent correlations between baseline skin and joint disease measures. ${ }^{8-11}$ Determining the relationship between severity of skin disease and joint disease activity is important for better phenotyping of patients with both skin and joint involvement and can guide clinicians in management. The objectives of this study were to compare patient characteristics (demographics, comorbidities, symptoms and medications) at enrolment among patient groups defined by degree of skin and joint involvement, and to evaluate the relationship between the degree of skin and joint involvement.

\section{METHODS}

\section{Data source}

The Corrona Registry is an independent, prospective, observational cohort of patients with PsA recruited at 40 private and academic practice sites across 25 states in the USA. ${ }^{12}$ As of 31 March 2018, the Corrona Psoriatic Arthritis/Spondyloarthritis (PsA/SpA) Registry database included information on 2827 patients. Data on 11525 patient visits and approximately 6278 patient-years of follow-up observation time had been collected. The mean time of patient follow-up was 3.1 years (median, 3.5 years). Data were collected from both patients and their treating rheumatologists, who gathered information on disease duration, prognosis, disease severity and activity, medical comorbidities, use of medications including biologics, conventional synthetic disease-modifying antirheumatic drugs (csDMARD), prednisone and adverse events. Follow-up assessments were requested at least as often as every 6 months and completed during routine clinical encounters. The Corrona Registry was established in accordance with the Declaration of Helsinki. All participating investigators were required to obtain full board approval for conducting non-interventional research involving human subjects. Sponsor approval and continuing review were obtained through a central Institutional Review Board (IRB). For academic investigative sites that did not receive a waiver to use the central IRB, full board approval was obtained from the respective governing IRBs and documentation of approval was submitted to Corrona before initiating any study procedures. All registry patients were required to provide written informed consent and authorisation before participating.

\section{Study population}

Our study cohort included 1542 patients in the Corrona PsA/SpA Registry who were continuously enrolled following an index date defined as enrolment between 21 March 2013 and 20 September 2016. Patients $\geq 18$ years of age with a diagnosis of PsA and with a history of $\mathrm{PsO}$ at the time of enrolment into the Corrona PsA/SpA Registry were recruited for the study. Patients were further required to have available body surface area (BSA) and Clinical Disease Activity Index (CDAI) measurements at enrolment. BSA is usually measured by estimating the per cent body involvement of $\mathrm{PsO}$, and it ranges from $0 \%$ to $100 \%$. One per cent BSA represents a one handprint size of the patient. The CDAI was developed to determine a patient' $s$ joint disease activity for rheumatoid arthritis but can also be used for PsA. ${ }^{13}$ The CDAI comprised four assessment tools: swollen (28) joint count, tender (28) joint count, Patient Global disease Activity and the Evaluator's Global disease Activity indexes. A CDAI activity score of $\leq 2.8$ is considered remission, a CDAI score $>2.8$ and $\leq 10$ is low disease activity, a CDAI score $>10$ and $\leq 22$ is moderate disease activity and a CDAI score $>22$ is high disease activity. ${ }^{14}$

For purposes of our study, patients with PsA were stratified by skin disease severity and joint disease activity determined at enrolment as follows: BSA $0 \%$ (history of $\mathrm{PsO}$ but not current/active); low (BSA $>0 \%$ to $\leq 3 \%$ ); and moderate-high (BSA $>3 \%$ ). Joint disease activity was measured by CDAI, where a CDAI $\leq 10$ was low and a CDAI $>10$ was considered moderate-high. Patients were further divided into six subgroups: BSA $0 \% /$ CDAI low; BSA $0 \% / \mathrm{CDAI}$ moderate-high; $\mathrm{BSA}>0 \%$ to $\leq 3 \% / \mathrm{CDAI}$ low; $\mathrm{BSA}>0 \%$ to $\leq 3 \% / \mathrm{CDAI}$ moderate-high; BSA >3\%/CDAI low; and BSA >3\%/ CDAI moderate-high. Demographic characteristics, duration of PsA, time since PsO diagnosis, clinical characteristics, patient-reported outcomes, history of PsA drug therapy and current PsA drug therapy at enrolment were also collected.

\section{Statistical analyses}

Comparison of baseline characteristics by skin disease severity and joint disease activity

Patient characteristics, including current and prior PsA medication use, were obtained during the enrolment visit and stratified by skin disease severity and joint disease activity. We compared baseline patient characteristics, clinical and disease characteristics and patient-reported factors among the six stratified skin disease severity-joint disease activity groups using appropriate methods: analysis of variance for continuous variables and $\chi^{2}$ test of association (for categorical variables; Fisher's test used if a cell count had $<5$ ). Any comparison with a resulting $p$ value $<0.05$ was flagged and considered in the list of factors used for evaluating the relationship between skin disease severity and joint disease activity.

Evaluating the relationship between skin disease severity and joint disease activity and factors that modify the relationship

The overall relationship of skin disease severity evaluated by BSA and joint disease activity (evaluated by CDAI) levels was calculated using the $\chi^{2}$ test of association, and the strength of the relationship between these two variables was measured using Cramer's V, which is the standard correlation of two categorical variables with two or more categories each. Cramer's V is used in conjunction with a significant $\chi^{2}$ test to determine the strength of association. Cramer's V ranges from 0 (no relationship) to 1 (strong relationship). Also, we calculated the OR 
to estimate the risk of being in moderate or high joint disease activity among patients with severe skin severity compared with patients with low joint disease activity and no skin involvement $(\mathrm{BSA}=0)$.

To further evaluate whether other variables (chosen a priori or identified through significance testing) modified the relationship between CDAI and BSA, multiple linear regression was performed starting with the list of a priori variables (ie, age, gender, body mass index (BMI), work status, smoking status, duration of PsA, time since PsO diagnosis, prior biologic or targeted synthetic DMARD (tsDMARD) use, current biologics or tsDMARD use, prior csDMARD use, Spondyloarthritis Research Consortium of Canada (SPARCC) enthesitis and dactylitis count and minimal disease activity (MDA)), and each variable was included in a separate model of CDAI regressed onto BSA. Effect modification of each variable was evaluated by including an interaction term (BSA and the variable under evaluation) into the linear regression model and assessed for statistical significance with the likelihood ratio test $\mathrm{p}<0.05$.

\section{RESULTS}

There were 1542 patients who met the inclusion criteria. The skin activity groups were categorised as follows: $24 \%$ ( $0 \%$ BSA), $44 \%$ (low BSA) and $32 \%$ of patients had moderate/high BSA levels. The low CDAI group comprised $62 \%$ of all patients (table 1 ).

\section{All patient demographic characteristics}

The preponderance of patients were $(52.9 \%)$ females. The mean age for all patients was 53.7 years, and most patients in the study $(94.4 \%)$ were white, and $52.8 \%$ never smoked. More than $62 \%$ of all patients worked part-time or full time, and $8.9 \%$ of all patients were disabled. Nearly $80 \%$ of all patients had private insurance of some kind, and the mean BMI for all patients was 31.5 $( \pm 7.2)$. The median PsA duration since diagnosis was 9 years, and 18 years for $\mathrm{PsO}$. Over $62 \%$ of all patients reported a history of biologic use. For those patients currently reporting DMARD therapy, $17.9 \%$ were not on any DMARD therapy at enrolment, $29 \%$ were on methotrexate or another csDMARD and $53.1 \%$ were on biologics/tsDMARDs (table 1).

\section{Patient demographic characteristics by degree of skin and joint involvement}

At the time of enrolment, within each BSA group, higher BMIs were found among patients with higher CDAI, and the highest BMI $(33.1 \pm 7.7)$ was observed in patients in the BSA $>3 \% / \mathrm{CDAI}$ medium-high subgroup. Significant differences were observed for current therapy among the six subgroups. Of note, those patients in the BSA 0\%/CDAI low subgroup for skin disease severity and joint disease activity reported the highest use of any drug therapy with $63.7 \%$ using biologics/tsDMARDs (table 1).
All patient disease characteristics and patient-reported outcomes

Among all patients, $18.3 \%$ had enthesitis and a SPARCC score $>0$. The mean SPARCC score for those with enthesitis was $4.1 \pm 3.1$. Nearly $45 \%$ of patients were in MDA. The mean BSA was $5.6( \pm 11.2)$, and the mean CDAI was $10.4( \pm 9.3)$. For the global assessment of $\mathrm{PsO}$, $25 \%$ were clear, $35.5 \%$ were almost clear, $24.4 \%$ had mild disease, $13.1 \%$ moderate disease and $2 \%$ severe disease. For the Disease Activity Score 28-joint count C-reactive protein, the majority of patients were in remission/low at $57.9 \%, 30.4 \%$ were moderate and $11.7 \%$ were considered high. The mean Nail PsO visual analogue scale (VAS) score was $7.2( \pm 16.5)$, and the mean Health Assessment Questionnaire (HAQ) was $0.61( \pm 0.6)$. Patient-reported pain was $37.6( \pm 29.3)$, and patient-reported fatigue was $40.2( \pm 29.1)$ (table 2$)$.

\section{Patient disease characteristics and patient-reported outcomes by degree of skin and joint involvement}

Patients in the BSA $>3 \% /$ CDAI moderate-high subgroup had the highest Nail PsO VAS with a median score of 17.5 $( \pm 23.4)$. For all patients, the highest mean HAQ of 1.06 $( \pm 0.7)$ was among patients in the BSA $>3 \% / \mathrm{CDAI}$ moderate-high subgroup. As BSA increased, the HAQ scores in the moderate-high CDAI groups also increased. In those patients with enthesitis, the highest SPARCC score of 5.3 $( \pm 3.1)$ was in the BSA 0\%/CDAI moderate-high subgroup. Patient-reported pain $(57.1 \pm 27.6)$ and patient-reported fatigue (54.0 28.2) are the highest among patients in the BSA $>3 \% / \mathrm{CDAI}$ moderate-high subgroup. Patients with PsA in the BSA 0\%/low CDAI subgroup had the highest mean EuroQol Group 5-Dimensional Questionnaire scores $(79.4 \pm 18.9)$ and a larger percentage $(18.3 \%)$ of patients reported no morning stiffness (table 2).

\section{The relationship between degree of skin and joint involvement}

Table 3 displays the classification table of skin disease severity (BSA, $0 \%,>0 \%$ to $3 \%,>3 \%$ ) by joint involvement (CDAI, low vs moderate-high). We observed a significant $(\mathrm{p}<0.001)$ modest association (Cramer's $\mathrm{V}=0.1639$ ) between skin severity and joint involvement among all patients at the time of enrolment in the study. Patients with higher skin disease severity were over two times more likely to have higher joint involvement (OR 2.27, 95\% CI 1.71 to 3.01). Significant levels of association between skin disease severity and joint disease activity were also observed when stratified by therapy status at the time of enrolment. The level of association among patients on no DMARD therapy (Cramer's V=0.2477) was higher compared with patients on csDMARDs only (Cramer's V=0.1439) or biologics or tsDMARDs (Cramer's $\mathrm{V}=0.1175$ ). Specifically, patients on no DMARD therapy at enrolment with higher skin disease severity were 4.5 times more likely to have higher joint involvement (OR 4.52, 95\% CI 2.10 to 9.73) whereas patients on biologics or tsDMARDs at enrolment with higher 


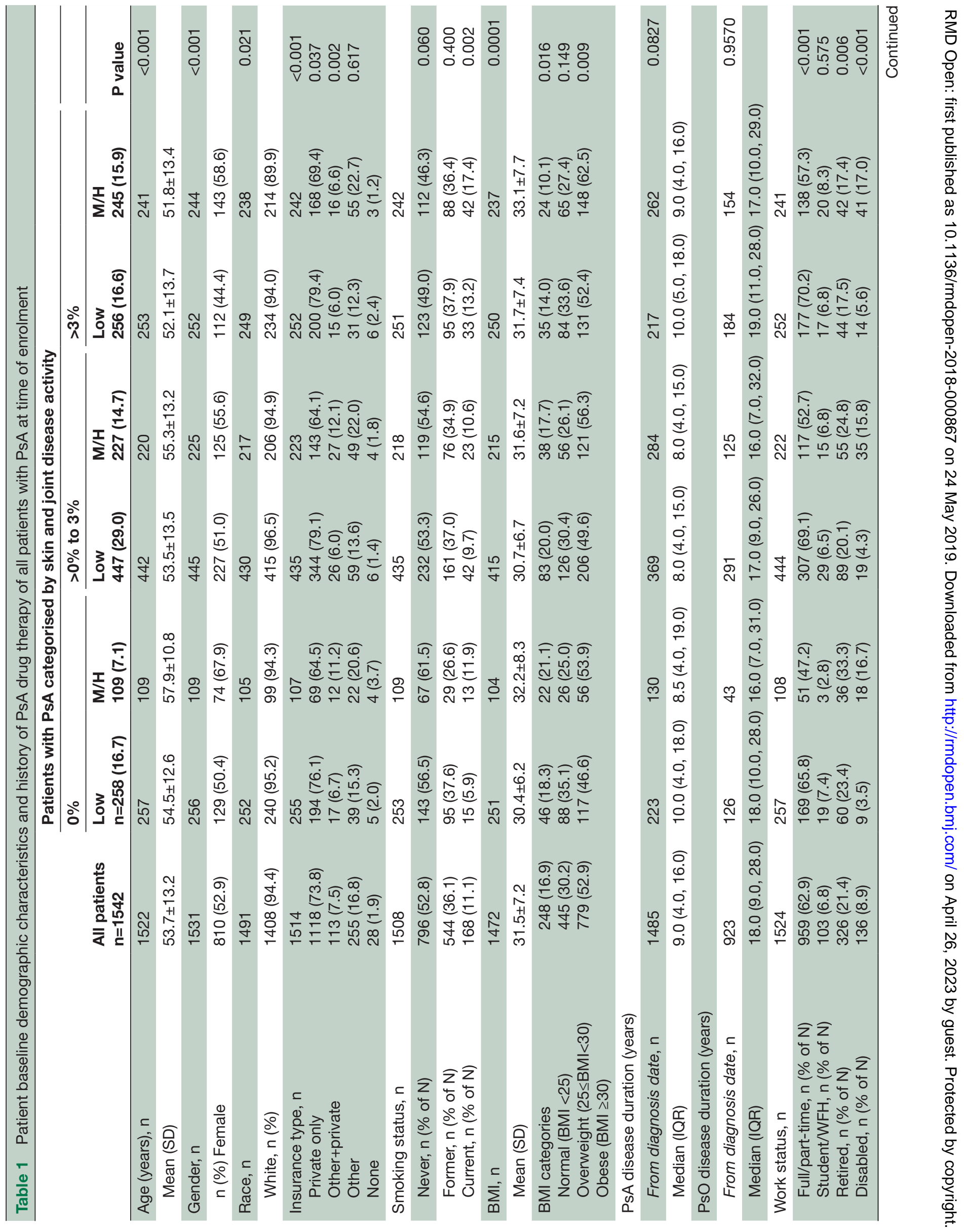




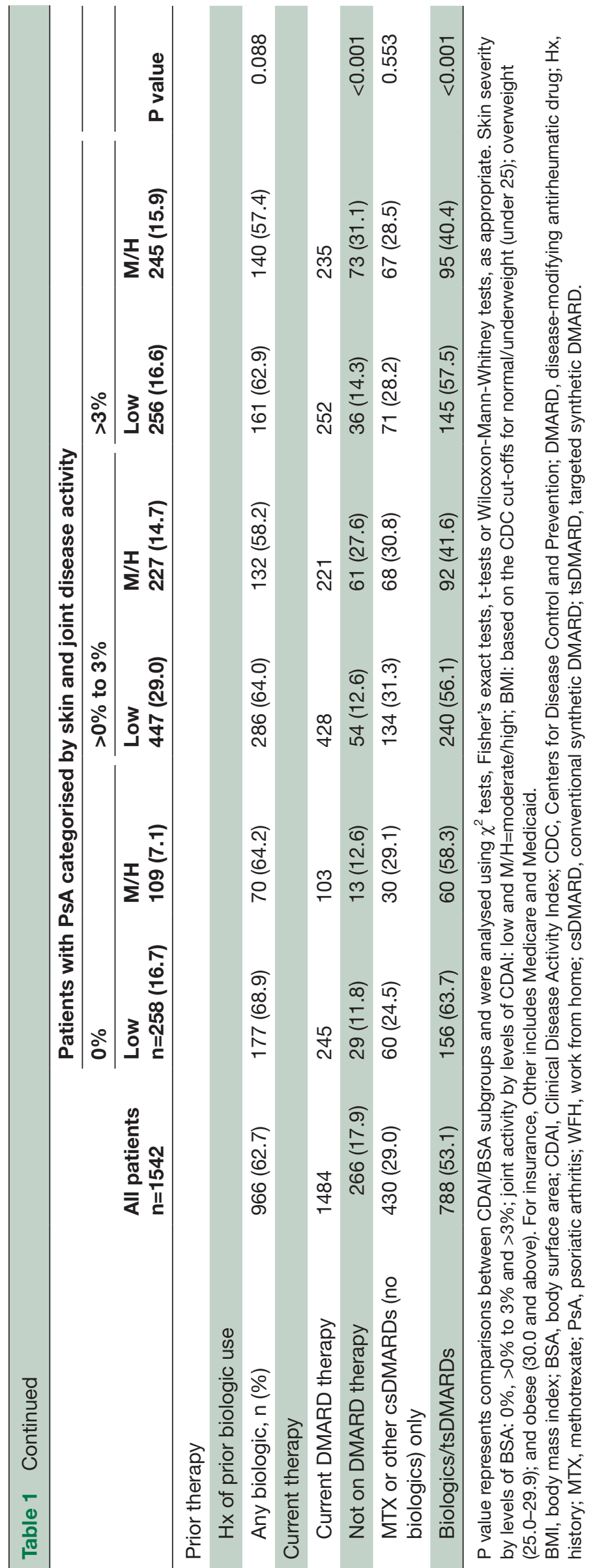




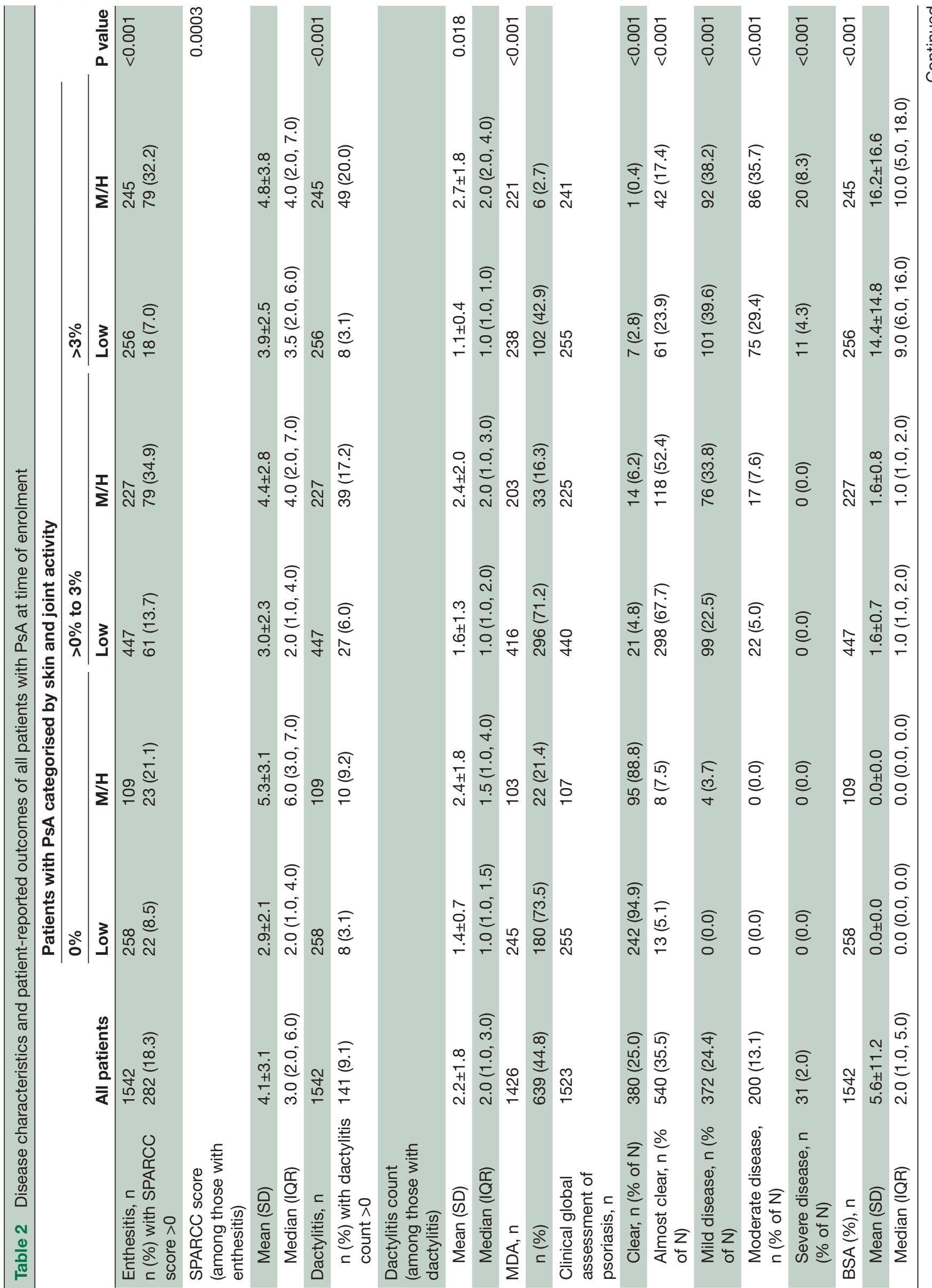




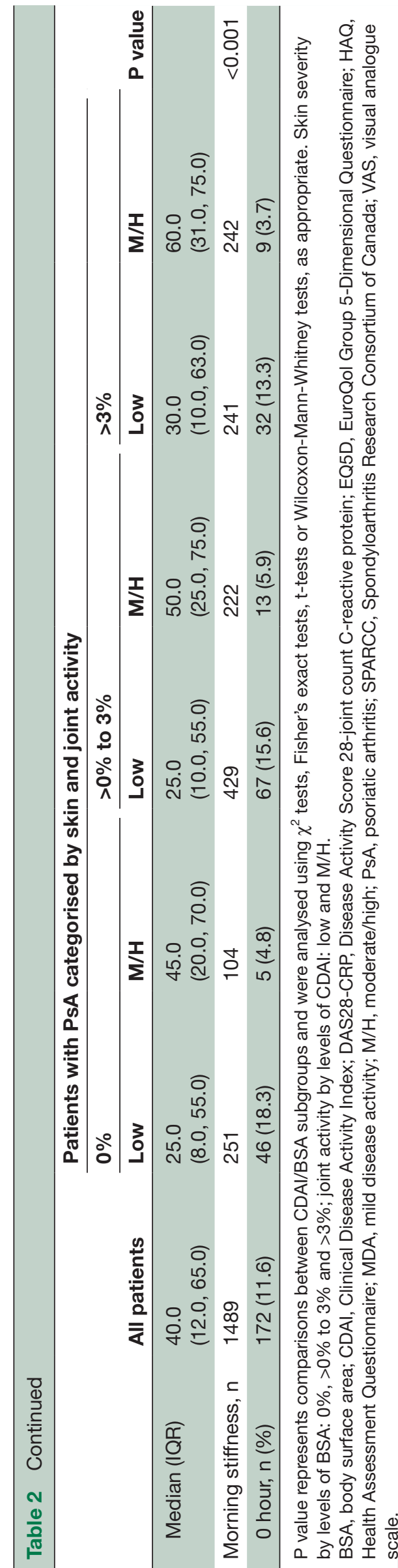

skin disease severity were 1.7 times more likely to have higher joint involvement (OR 1.70, 95\% CI 1.15 to 2.52).

We observed a significant linear relationship between CDAI and BSA (linear regression of CDAI onto BSA is $\mathrm{CDAI}=9.48+0.16 * \mathrm{BSA}$ (95\% CI 0.12 to 0.20 for $\mathrm{BSA}$ coefficient). When we evaluated the linear relationship of CDAI and BSA accounting for a priori selected factors as potential effect modifiers, we observed significant interactions (evidence of effect modification) that showed this relationship was modified when accounting for other factors. Figure 1 provides a visual representation of the effect modification between CDAI and BSA by age, gender, work status, insurance, current therapy at enrolment and levels of HAQ, Nail VAS, MDA, dactylitis total, patient-reported pain and patient-reported fatigue. The red vertical line $(y=0.16)$ represents the 0.16 unit increase in CDAI for every unit increase in BSA (from the linear regression of CDAI onto BSA). Deviations from this vertical line indicate how different levels of the identified factors modify the association between CDAI and BSA such that a stronger positive association is observed (point estimate of slope coefficient for BSA within the subgroup defined by covariate $>0.16$ ), weaker positive association (point estimate of slope coefficient for BSA within the subgroup defined by covariate $<0.16$ ) or negative association (point estimate of slope coefficient for BSA within the subgroup defined by covariate $<0)$. The blue dotted vertical line $(y=0)$ represents no association.

We observed a weaker relationship between CDAI (point estimate $<0.16$ ) and BSA when a patient appeared to have their disease under control. These patients were on some type of biologic or tsDMARD therapy, either younger (18-30 years) or older ( $>50$ years), were in MDA, had no nail involvement (Nail VAS $=0$ ) or did not have dactylitis. There was an inverse relationship (point estimate of slope coefficient for BSA within the subgroup $<0)$ between CDAI and BSA among patients with lowest reported pain (0-20) and fatigue (0-20), or had low levels of HAQ; however, the strength of the positive relationship between CDAI and BSA increased as pain, fatigue and HAQ levels increased, respectively. A weaker relationship (point estimate of slope coefficient for BSA within the subgroup $<0.16$ ) between CDAI and BSA was observed in males compared with females. A stronger relationship (point estimate of slope coefficient for BSA within the subgroup $>0.16$ ) was found among patients on Other (Medicare or Medicaid) or no insurance compared with patients on some type of private insurance (with or without Medicare or Medicaid). When we considered work status, we observed that for every unit increase in BSA, CDAI had, on average, a unit increase of $>0.16$ for students and those patients with PsA who work at home or were disabled, while the mean unit increase of CDAI was $<0.16$ for retirees. 
Table 3 The classification of skin disease severity (0\%, >0\% to $3 \%,>3 \%)$ by joint involvement (low vs moderate-high) BSA

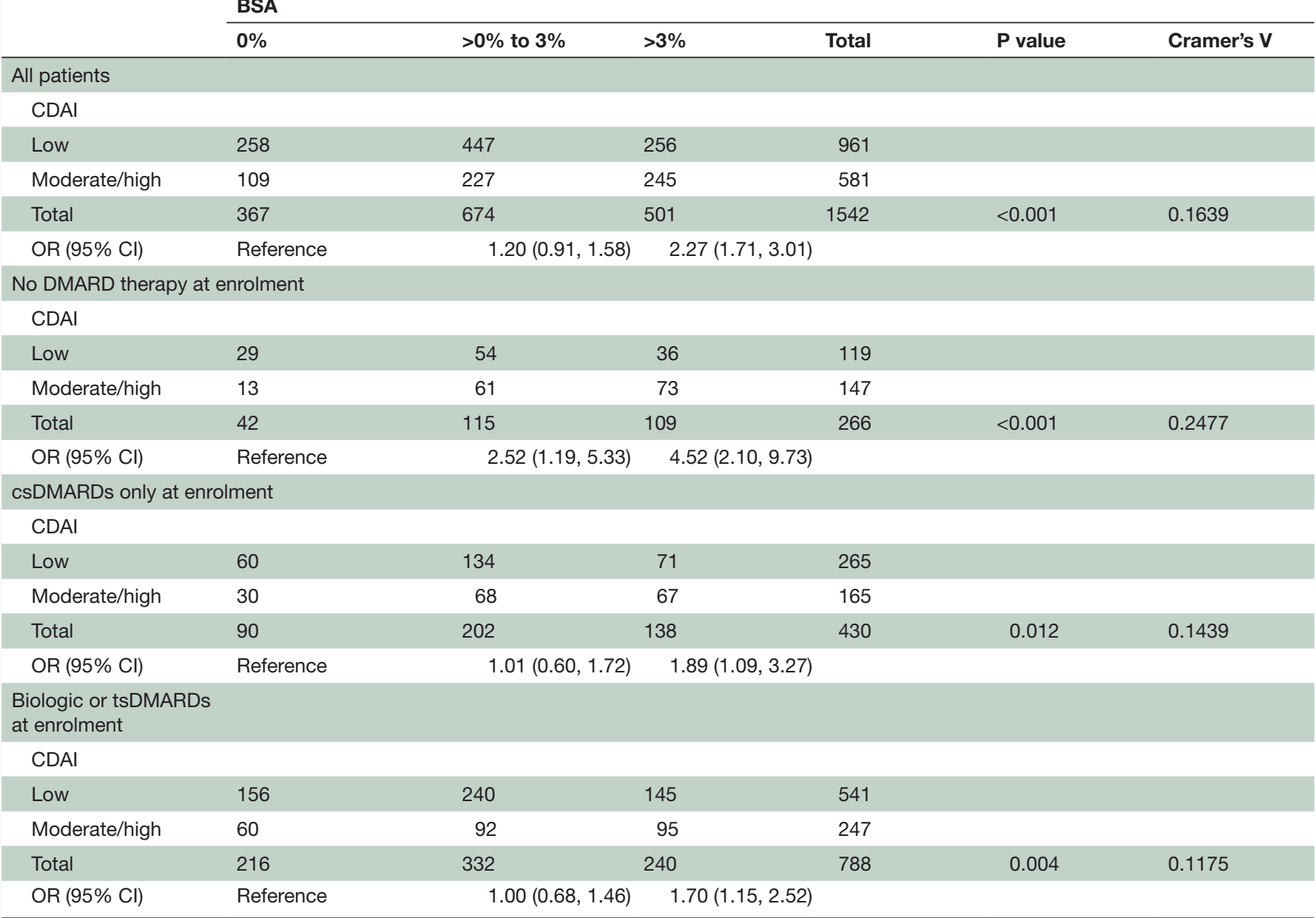

Drug therapy was missing for 58 patients. $P$ value for $\chi^{2}$ test of association.

BSA, body surface area; CDAl, Clinical Disease Activity Index; DMARD, disease-modifying antirheumatic drug; OR, odds ratio; csDMARD, conventional synthetic DMARD; tsDMARD, targeted synthetic DMARD.

\section{DISCUSSION}

Our objective was to compare patient characteristics at enrolment among patient groups stratified by degree of skin and joint involvement and to evaluate the relationship between degree of skin and joint involvement in patients with PsA. Our study found substantial variation of skin and joint involvement in this cohort of patients with PsA, with some experiencing more articular versus skin disease and vice versa. Current therapy at enrolment was also significantly different across the skin and joint subgroups. It is important to note that most patients in the BSA $>3 \% / \mathrm{CDAI}$ moderate-high subgroup had worse clinical and patient-reported outcomes. Moreover, those patients in the BSA 0\%/CDAI low subgroup reported the highest use of any drug therapy with $63.7 \%$ using biologics/tsDMARD. This may speak to the effectiveness of such therapies.

We also observed a statistically significant association between skin disease severity and joint involvement among all patients at the time of enrolment in the study. Furthermore, when we evaluated the linear relationship of CDAI and BSA, we found that some factors (ie, age, gender, insurance, work status, current therapy, HAQ, Nail VAS, MDA, dactylitis count, patient-reported pain and patient-reported fatigue) modified this relationship. In evaluating the association between CDAI and BSA (categorised by severity level), we observed that the association was strongest among patients on no therapy, followed by patients on csDMARD therapy, versus biologic or tsDMARD therapy. However, when evaluating the linear association between CDAI and BSA (in their continuous form), the linear association was strongest within the csDMARD therapy group while the association within the no therapy group is lower compared with the csDMARD group. We account for this difference due to the nature of the analysis (linear association of two continuous vs association between two categorical variables). In the evaluation of the linear association, the magnitude of the CDAI and BSA values (especially those in higher levels of activity) will affect the estimate of the association, whereas the effects of these larger in magnitude values are tempered when categorised into a high severity group. 


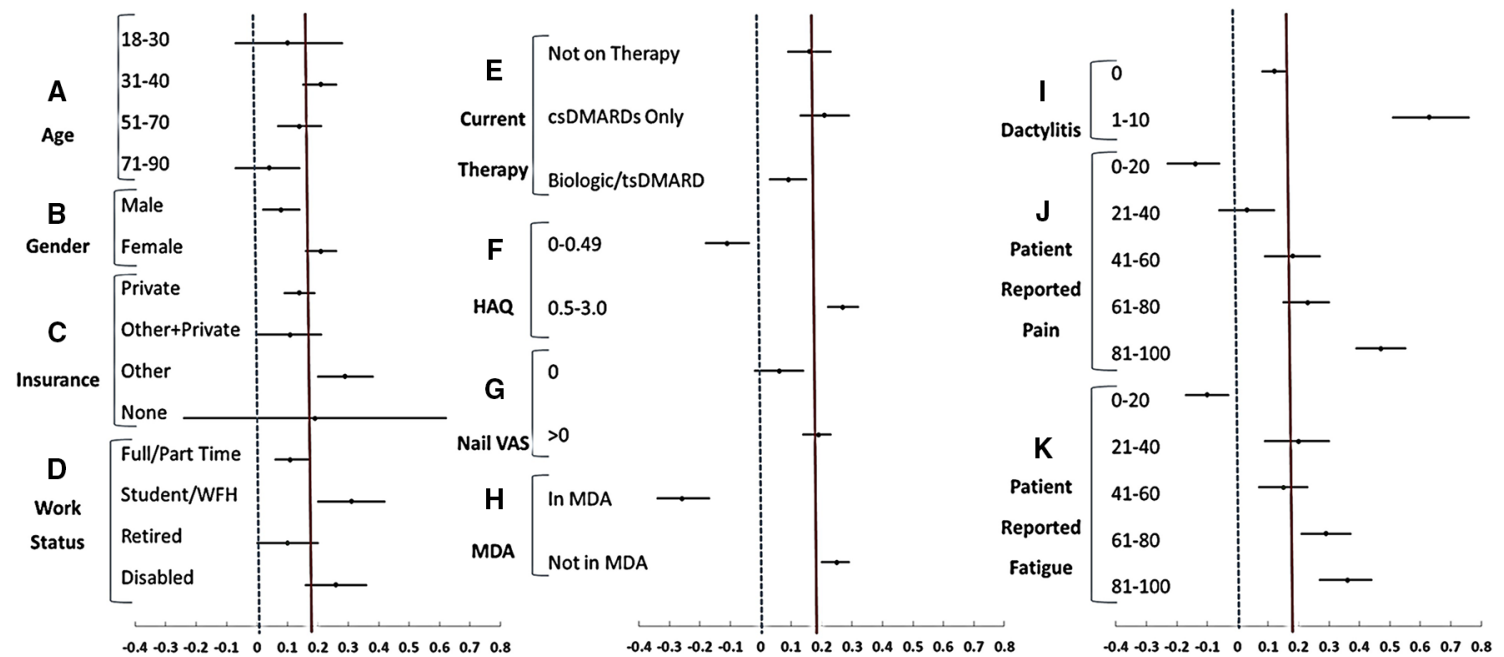

Our study found a statistically significant association between joint disease activity and skin disease severity. Specifically, patients with higher skin disease severity (BSA >3\%) were two times more likely to have higher joint involvement compared with those with a $\mathrm{BSA}=0 \%$. These findings underscore that skin disease severity and joint disease activity are related and addressing both manifestations is essential in caring for patients with PsA.

Our findings of an increased likelihood of higher joint involvement with high skin severity suggest the need for rheumatologists and dermatologists to approach PsA patient care comprehensively. The relationship between skin and joint severity identified in our study indicates that in those patients who have a positive articular response to therapy, rheumatologists need to assess whether there is increasing skin involvement to ensure adequate control of both joint and skin symptoms. Likewise, when skin disease is well managed by dermatologists, but joint disease symptoms are persistent, rheumatologic consultation should be pursued. Although previous studies have reported an association only between joint activity and nail involvement, ${ }^{910}$ our study further showed the association between skin disease severity and joint disease activity was stronger in certain patient subgroups, namely those who were not in MDA, with higher Nail VAS severity, in patients who worked from home or were students, and who had higher HAQ and other patient-reported outcome scores. Because of the exploratory nature of our study, more investigation is needed to confirm these observations.
Some limitations should be noted. In this study, we used CDAI (based on 28 tender and swollen joint counts) to define joint disease activity. Although initially derived for rheumatoid arthritis, CDAI has been used to measure joint activity in patients with PsA. However, a limitation of using CDAI is that it does not capture articular involvement of feet and ankle which is frequent in PsA. The Disease Activity in Psoriatic Arthritis (DAPSA) score includes 66/68 joint counts, C-reactive protein (CRP) as well as patient pain assessment. By using CDAI instead of DAPSA, we were able to achieve a larger sample size since CRP is not a required reporting measure in the Corrona Registry and not available for all patients. The cDAPSA score (DAPSA with the removal of CRP) includes patient pain assessment, and if we used CDAPSA, another consideration, we could not have evaluated the modification of the association between joint activity and skin severity by patient pain, a goal of our study. Moreover, we evaluated the correlation between CDAI and cDAPSA and observed a high correlation $(0.78)$ between these two disease activity measures in our study. Misclassification due to recall bias is a potential limitation since the registry is not based on an inception cohort and patients could enter the study at any time during the course of their disease. The registry data are from a US-based registry, and the results may not be generalisable to populations residing outside the USA. In our study, the majority of patients $(94.4 \%)$ were white, and thus the results may not be applicable to other racial backgrounds. However, the strengths of this study include the ability to identify a sufficiently large cohort of patients with PsA to characterise the relationship 
between skin disease severity and joint disease activity, as well as to identify patient characteristics that modify that relationship.

Our findings demonstrate that skin disease severity is modestly correlated with joint disease activity, the relationship is affected by several patient and disease factors and those patients with higher skin disease severity are two times more likely to have higher joint involvement. Understanding the association between skin and joint involvement among patients with PsA emphasises the need for providers (specifically rheumatologists and dermatologists) to consider both skin disease severity and joint disease activity in the treatment of these patients. Further investigation of the association will help define gaps in current therapy regimens, such as an inherent tendency to focus on just one of the clinical domains of the disease (ie, skin or joint disease), not both; and address the clinical inertia by both providers and patients as it relates to the level of baseline expectations and to the satisfaction with treatment. Studying these differences will help us understand to what extent in the real world skin severity and joint activity correlate.

\section{Author affiliations}

${ }^{1}$ Swedish Rheumatology Research Group, Swedish Medical Center and University of Washington, Seattle, Washington, USA

${ }^{2}$ Corrona, Waltham, Massachusetts, USA

${ }^{3}$ Department of Epidemiology, UT MD Anderson Cancer Center, Houston, Texas, USA ${ }^{4}$ Eli Lilly and Company, Indianapolis, Indiana, USA

${ }^{5}$ Department of Dermatology, Keck School of Medicine, University of Southern California, Los Angeles, California, USA

${ }^{6}$ New York University Hospital for Joint Diseases, New York City, New York, USA

Acknowledgements Michelle D Karpman, $\mathrm{PhD}$ (Corrona), provided scientific writing support.

Contributors All authors have made substantial contributions to the intellectual content of the manuscript: conception of the work; design of the work; acquisition of data for the work; analysis of data for the work; interpretation of data for the work; drafting of the manuscript; critical revision of the manuscript for important intellectual content.

Funding The study was sponsored by Corrona. Corrona has been supported through contracted subscriptions in the last two years by AbbVie, Amgen, Boehringer Ingelheim, Bristol-Myers Squibb, Crescendo, Eli Lilly, Genentech, Gilead, GSK, Horizon Pharma USA, Janssen, Merck, Momenta Pharmaceuticals, Novartis, Pfizer, Roche, UCB and Valeant.

Competing interests PJM: research grants: AbbVie, Amgen, BMS, Celgene, Janssen, Lilly, Novartis, Pfizer, Sun, UCB; consulting: AbbVie, Amgen, BMS, Celgene, Janssen, Lilly, Novartis, Pfizer, Sun, UCB; speakers' bureau: AbbVie, Amgen, BMS, Celgene, Genentech, Janssen, Novartis, Pfizer, UCB. CJE: employee at Corrona and advisory board for Merck. WJH, TMM, JRL, MJM and WNM: employees and stockholders at Eli Lilly. AWA has served as investigator, advisor and/or consultant to AbbVie, Janssen, Novartis, Lilly, Regeneron, Sanofi, Leo, Science 37, Modernizing Medicine and Ortho Dermatologics. SR and RD: employees at Corrona. JDG: employee and shareholder at Corrona; consultant for Genentech, Janssen, Novartis, Pfizer and Eli Lilly.

Patient consent for publication Not required.

Ethics approval Sponsor approval was obtained through a central Institutional Review Board (IRB). For academic investigative sites that did not receive a waiver to use the central IRB, full board approval was obtained from the respective governing IRBs and documentation of approval was submitted to Corrona before initiating any study procedures.

Provenance and peer review Not commissioned; externally peer reviewed.

Data sharing statement Data may be obtained from a third party and are not publicly available.

Open access This is an open access article distributed in accordance with the Creative Commons Attribution Non Commercial (CC BY-NC 4.0) license, which permits others to distribute, remix, adapt, build upon this work non-commercially, and license their derivative works on different terms, provided the original work is properly cited, appropriate credit is given, any changes made indicated, and the use is non-commercial. See: http://creativecommons.org/licenses/by-nc/4.0/.

\section{REFERENCES}

1. Lloyd P, Ryan C, Menter A. Psoriatic arthritis: an update. Arthritis 2012;2012:1-6.

2. Gladman DDet al. Psoriatic arthritis: epidemiology, clinical features, course, and outcome. Annals of the Rheumatic Diseases 2005;64(suppl_2):ii14-17.

3. Gottlieb Aet al. Guidelines of care for the management of psoriasis and psoriatic arthritis: Section 2. Psoriatic arthritis: overview and guidelines of care for treatment with an emphasis on the biologics. $J$ Am Acad Dematol 2008;56:851-64.

4. Rachakonda TD, Schupp CW, Armstrong AW. Psoriasis prevalence among adults in the United States. Journal of the American Academy of Dermatology 2014;70:512-6.

5. Mease PJ, Gladman DD, Papp KA, et al. Prevalence of rheumatologist-diagnosed psoriatic arthritis in patients with psoriasis in European/North American dermatology clinics. Journal of the American Academy of Dermatology 2013;69:729-35.

6. Rosen CF, Mussani F, Chandran V, et al. Patients with psoriatic arthritis have worse quality of life than those with psoriasis alone. Rheumatology 2012;51:571-6.

7. Feldman SR, Zhao Y, Shi L, et al. Economic and comorbidity burden among moderate-to-severe psoriasis patients with comorbid psoriatic arthritis. Arthritis Care \& Research 2015;67:708-17.

8. Gottlieb AB, Mease PJ, Mark Jackson J, et al. Clinical characteristics of psoriatic arthritis and psoriasis in dermatologists' offices. Journal of Dermatological Treatment 2006;17:279-87.

9. Jones SM, Armas JB, Cohen MG, et al. Psoriatic arthritis: outcome of disease subsets and relationship of joint disease to nail and skin disease. Rheumatology 1994;33:834-9.

10. Cohen MR, Reda DJ, Clegg DO. Baseline relationships between psoriasis and psoriatic arthritis: analysis of 221 patients with active psoriatic arthritis. Department of veterans affairs cooperative study group on seronegative spondyloarthropathies. J Rheumatol 1999;26:1752-6.

11. Elkayam O, Ophir J, Yaron M, et al. Psoriatic arthritis: Interrelationships between skin and joint manifestations related to onset, course and distribution. Clin Rheumatol 2000;19:301-5.

12. Kremer JM. The CORRONA database. Clin Exp Rheumatol 2005;23(5 Suppl 39):iv37-41.

13. Acosta Felquer ML, Ferreyra Garrott L, Marin J, et al. Remission criteria and activity indices in psoriatic arthritis. Clin Rheumatol 2014;33:1323-30.

14. Anderson JK, Zimmerman L, Caplan L, et al. Measures of rheumatoid arthritis disease activity: patient (PtGA) and provider (PrGA) global assessment of disease activity, disease activity score (DAS) and disease activity score with 28-Joint counts (DAS28), simplified disease activity index (SDAI), clinical disease activity index (CDAl), patient activity score (PAS) and patient activity Score-II (PASII), routine assessment of patient index data (Rapid), rheumatoid arthritis disease activity index (radai) and rheumatoid arthritis disease activity Index-5 (RADAI-5), chronic arthritis systemic index (CASI), patient-based disease activity score with ESR (PDAS1) and patient-based disease activity score without ESR (PDAS2), and mean overall index for rheumatoid arthritis (MOI-RA). Arthritis Care Res 2011;63 Suppl 11:S14-S36. 